\title{
Expression and functional analysis of Brucella outer membrane protein 25 in recombinant goat pox virus
}

\author{
ZHIHUA SUN ${ }^{1 *}$, LAIZHEN LIU ${ }^{1 *}$, HUI ZHANG ${ }^{1}$, YOUWEN LI ${ }^{1}$, FENG WEI $^{1}$, ZHIQIANG LI $^{2}$, \\ PENGYAN WANG $^{1}$, QIANG FU ${ }^{1}$, YAN REN $^{3}$, YU ZHANG $^{1}$, ZHIRU GUO $^{1}$ and CHUANGFU CHEN ${ }^{1}$ \\ ${ }^{1}$ College of Animal Science and Technology, Shihezi University, Shihezi, Xinjiang 832000; \\ ${ }^{2}$ College of Biology and Food, Shangqiu Normal University, Shangqiu, Henan 476000; \\ ${ }^{3}$ College of Medicine, Shihezi University, Shihezi, Xinjiang 832000, P.R. China
}

Received August 26, 2016; Accepted May 22, 2017

DOI: $10.3892 / \mathrm{mmr} .2019 .9868$

\begin{abstract}
The Capripoxvirus (CaPV) has a large double-stranded DNA genome and a restricted host-range. At present, it is being investigated as an ideal vaccine vector. In the present study, a novel recombinant goat pox virus (rGTPV) was constructed to express Brucella outer membrane protein (OMP)25, and was validated by in vitro and in vivo immunization assays. A novel rGTPV vector was created, in which the thymidine kinase gene was used as a flanking sequence, $\mathrm{I} 1 \mathrm{~L}$ was inserted as a promoter element to enhance Brucella OMP25 expression, and p7.5 as another promoter element was used to regulate guanine phosphoribosyl-transferase as a selection maker. The rGTPV vector was transfected into sheep fetal fibroblast/lamb testis cells pre-infected with GTPV G14-STV44-55 to recombine. Brucella OMP25 protein was expressed in cells by rGTPV, and activated immune reactivity to Brucella OMP25 protein, as detected by western blotting. Furthermore, rGTPV elicited, anti-Brucella-specific immunoglobulin G responses, as determined by ELISA. Mice vaccinated with rGTPV did not exhibit pathology alterations in the kidney and liver. These results suggested that the novel rGTPV was able to efficiently drive Brucella OMP25 protein expression and activate immune reactivity, and may have applications in $\mathrm{CaPV}$ live vector vaccines and associated research.
\end{abstract}

Correspondence to: Professor Hui Zhang or Professor Chuangfu Chen, College of Animal Science and Technology, Shihezi University, 221 Bei 4th Road, Shihezi, Xinjiang 832000, P.R. China

E-mail: allanzhh@sohu.com

E-mail: ccf_xb@126.com

*Contributed equally

Key words: Brucella, goat pox virus, promoter, recombinant goat pox virus-outer membrane protein 25

\section{Introduction}

Brucella spp. are facultative intracellular bacteria that cause brucellosis, which is a globally occurring zoonotic disease (1). The disease is characterized by abortion in domestic animals, and undulant fever, arthritis, endocarditis and meningitis in humans (2). Brucella normally colonize the host reticuloendothelial system and occasionally other target organs, including the joints and placenta (3). There are currently no licensed Brucella vaccines for human use, and only a few licensed live Brucella vaccines are available for use in animals. However, the available animal vaccines may cause abortion and are associated with lower protection rates in animals and higher virulence in humans. An increasing amount of research has been recently performed to develop novel Brucella vaccines for the prevention and control of animal brucellosis.

Vaccination is a crucial component of brucellosis eradication programs worldwide (4). Efforts to develop an effective, stable and non-reactogenic vaccine against brucellosis have been ongoing in a number of laboratories, and the use of a live, attenuated platform has become the established benchmark through the use of the B. abortus rough strain RB51 (5). To improve the safety of the vaccine, researchers have conducted studies of genetically engineered vaccines, particularly recombinant live vector vaccines. Poxvirus is a widely used live vector (6); however, it may additionally infect specific types of animals, including humans, thus Capripoxvirus (CaPV) are now more frequently used because of their more restricted host specificity. The family Poxviridae includes numerous viruses of medical and veterinary importance. Goat pox virus (GTPV) is member of the CaPV genus of the Poxviridae, and causes the disease of goat pox (7). The genome is $150 \mathrm{kbp}$ double-stranded DNA, which encodes at least 147 open reading frames, including conserved replicative and structural genes, and genes that are likely involved in virulence and host range (8). The GTPV is an ideal recombinant live vaccine vector for the principal infectious diseases of sheep. To select a recombinant virus, researchers have taken advantage of the recent characterization of the vaccinia virus thymidine kinase (TK) gene (9). Therefore, the present study aimed to construct plasmids containing a Brucella protective antigen inserted within the vaccinia virus TK gene. 
I1L is a promoter of GTPV that is highly active. The activity of the I1L promoter may be amenable to high-level expression of target genes. This promoter is a useful tool for poxvirus expression and does not require a specialized virus expressing a heterologous protein (10).

Therefore, the present study used intergenic regions around the central genomic region of GTPV for the insertion of foreign genes, based on the highly homologous genomic structures of the CaPVs. These regions were used to insert the guanine phosphoribosyl-transferase (GPT) gene of Escherichia coli (E. coli) as a dominant selectable marker (11). In the present study, a recombinant (r)GTPV vector was used, which selected the TK gene as an insertion site for an exogenous gene and the vaccine virus I1L promoter to regulate the expression of a foreign gene. As a result, the Brucella outer membrane protein (OMP)25 was expressed. These foreign genes were successfully expressed and demonstrated immunogenicity.

\section{Materials and methods}

Ethics statement. The present study was approved by the Institutional Committee of Post-Graduate Studies and Research at Shihezi University (Shihezi, China). All efforts were made to minimize animal suffering.

Virus and cells. The GTPV strain G14-STV44-55, an attenuated live vaccine, was provided by Xinjiang Tecon Animal Husbandry Biotechnology Co., Ltd. (Urumqi, China). Sheep fetus fibroblast (SFF) cells and lamb testis (LT) cells (obtained from Cell Resource Center, Institute of Basic Medical Sciences, Chinese Academy of Medical Sciences, Beijing, China) were cultured in Dulbecco's modified Eagle's medium (DMEM; Sigma-Aldrich; Merck KGaA, Darmstadt, Germany) supplemented with $10 \%$ fetal bovine serum (FBS; Gibco; Thermo Fisher Scientific, Inc., Waltham, MA, USA) at $37^{\circ} \mathrm{C}$ in $5 \% \mathrm{CO}_{2}$. The GTPV was propagated and titrated on SFF or LT cells in DMEM supplemented with $2 \%$ FBS. The plasmid pLSEG-GPT includes the GPT gene and $7.5 \mathrm{~K}$ early/late promoter (P7.5). The plasmid pGM-TK-I1L-GPT1 was synthesized by the Key Laboratory of Xinjiang Endemic and Ethnic Disease, Shihezi University.

Mice.Intotal,30 female six-week-oldBALB/c mice (weight, $25 \mathrm{~g}$ ) were obtained from the Experimental Animal Center of the Academy Military Medical Science (Beijing, China). Animals were maintained in barrier housing with filtered inflow air in a restricted-access room in pathogen-limited conditions. The light/dark cycle was $12 / 12 \mathrm{~h}$, the temperature was $26^{\circ} \mathrm{C}$ and the humidity was $40-60 \%$. They were acclimatized for a minimum of 1 week prior to experimentation. Water and commercial food were provided ad libitum. All experimental procedures and animal care were performed in compliance with institutional animal care regulations.

Construction of the GTPV G14-STV44-55 strain expression vector and expression of B. melitensis OMP25 by rGTPV. Total genomic DNA was extracted from infected LT cells using a commercial virus DNA extraction kit (Bioteke Corporation, Beijing, China). Polymerase chain reaction (PCR) amplification of the conserved TK gene was successfully performed on all isolates using Taq DNA polymerase (Takara Biotechnology, Co., Ltd., Dalian, China) for PCR with specific primers (Table I). The PCR reaction system contained the following: $1.5 \mu \mathrm{l} 10 \mathrm{X}$ buffer, $0.2 \mu \mathrm{ldNTP}$ (10 mmol/1), $1 \mu \mathrm{l}$ DNA $(20 \mathrm{ng} / \mu \mathrm{l}), 0.2 \mu \mathrm{l}$ Taq enzyme, $0.2 \mu \mathrm{l}$ primers $(20 \mu \mathrm{mol} / \mathrm{l})$, $0.6 \mu 1 \mathrm{MgCl}_{2}$ (25 mmol/1; Tiangen Biotech Co., Ltd., Beijing, China) and $11.1 \mu 1 \mathrm{H}_{2} \mathrm{O}$. The total volume was $15 \mu \mathrm{l}$. The PCR reaction conditions were as follows: $5 \mathrm{~min}$ at $95^{\circ} \mathrm{C}$; followed by 30 cycles at $60^{\circ} \mathrm{C}$ for $40 \mathrm{sec}$ and $72^{\circ} \mathrm{C}$ for $1 \mathrm{~min}$; and $10 \mathrm{~min}$ at $72^{\circ} \mathrm{C}$. PCR products were analyzed on $1 \%$ agarose gels containing ethidium bromide under a UV transilluminator. Subsequently, PCR products were ligated to the plasmid pGEM-T (Tiangen Biotech, Co., Ltd.) for DNA sequencing.

Fick and Viljoen (12) previously identified the VVI1L promoter sequence; therefore, the present study used the reverse complementary sequence of the VVI1L promoter and the GPT gene promoter, overlapping synthesis of primers in the opposite direction (Table I).

The vaccinia virus p7.5 promoter that regulates xanthine-guanine GPT was obtained by XhoI/NotI digestion of plasmid pLSEG-GPT, and was used as a positive selection marker for recombinant virus screening. It was ligated to the XhoI and Not I restriction sites of pBS-T. This constructed element was inserted at the Acc65I restriction sites of the TK gene of strain G14-STV44-55, and was subsequently used for the construction of a transfer vector, which was termed pGM-TK-I1L-GPT.

The OMP25 DNA (642 bp) of Brucella was amplified with OMP25-F and OMP25-R, in which the Xho I sites products were ligated with pGM-TK-I1L-GPT vector to generate pGM-TK-I1L-GPT-OMP25.

Screening and identifying for recombinant virus. Confluent LT cells were co-transfected with GTPV G14-STV44-55 and the expression vector pGM-TK-I1L-GPT-omp25, using Lipofectamine ${ }^{\circledR} 2000$ (Invitrogen; Thermo Fisher Scientific, Inc.). Cultures were subsequently harvested when $80 \%$ cytopathic effect (CPE) was observed. Cultured LT cells were digested with $0.25 \%$ trypsin and resuspended in screening solution (DMEM, 2.5\% fetal calf serum (Gibco; Thermo Fisher Scientific, Inc.), $100 \mathrm{U} / \mathrm{ml}$ penicillin/streptomycin, $25 \mu \mathrm{g}$ MPA ml$l^{-1}, 250 \mu \mathrm{g}$ xanthine $\mathrm{ml}^{-1}, 15 \mu \mathrm{g}$ hypoxanthine $\mathrm{ml}^{-1}, 2 \mu \mathrm{g}$ aminopterin $\mathrm{ml}^{-1}$ and $2 \mu \mathrm{g}$ thymidine $\mathrm{ml}^{-1}$ ). Following centrifugation at $350 \mathrm{x} \mathrm{g}$ for $5 \mathrm{~min}$ at room temperature, cells were added to 12 -well cell culture plates

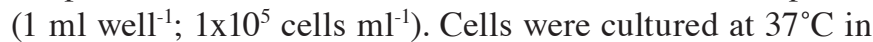
$5 \% \mathrm{CO}_{2}$ for $16-24 \mathrm{~h}$ until $70-80 \%$ confluent. Subsequently, cultures were harvested once they reached $80 \% \mathrm{CPE}$, and were inoculated on LT cells following repeated freezing and thawing three times. Cells were cultured at $37^{\circ} \mathrm{C}$ in $5 \% \mathrm{CO}_{2}$ for 14 days and observed once daily. The cytopathic culture medium was collected, and centrifuged at $350 \mathrm{x}$ g for $5 \mathrm{~min}$ at room temperature to remove cell debris, and used for further screening with this method. The recombinant virus was confirmed by PCR following the protocol mentioned above.

Electron microscopy. LT cells were infected with the recombinant virus. For transmission electron microscopy, at 14 days post-infection, cells were fixed with $4 \%$ glutaraldehyde for 
Table I. Primers for polymerase chain reaction.

\begin{tabular}{lll}
\hline Primer name & \multicolumn{1}{c}{ Sequence, 5'-3' } & Product size, bp \\
\hline TK-F & GAATTCCAAGCCACTACAAGAACCAGTTAG & 2,400 \\
TK-R & CTGCAGCTGATCCAACATATACTATTGTGC \\
I1L-GPT-F & CTCGAGCCATTTAAGTTCACCAAACAACTTTTA & 1,200 \\
I1L-GPT-R & AATAAGGCCTAATTAATTAAGTCG \\
OMP25-F & CTCGAGCTATATGGCCCGGTCCGGTTAACTA \\
OMP25-R & CTCGAGATGCTCACACTCTTAAGTCTC & 642 \\
\hline
\end{tabular}

Underlined nucleotides are restriction sites of the enzymes indicated in the brackets at the ends. F, forward; R, reverse; TK, thymidine kinase; GPT, guanine phosphoribosyl-transferase; OMP25, outer membrane protein 25.

$1 \mathrm{~h}$ at room temperature overnight at $4^{\circ} \mathrm{C}$, dehydrated in an ascending ethanol series and embedded for $1 \mathrm{~h}$ at room temperature in Epoxi resin (Epoxi Embedding Medium kit; Sigma-Aldrich; Merck KGaA). Ultrathin sections (45 nm) were stained with $2 \%$ uranyl acetate and Reynold solution for $1 \mathrm{~h}$ at room temperature. For electron microscopy, cells were fixed with $4 \%$ paraformaldehyde and $0.25 \%$ glutaraldehyde for $24 \mathrm{~h}$ at room temperature. Ultrathin sections were collected in nickel grids and observed using a Zeiss EM109T transmission electron microscope (Zeiss GmbH, Jena, Germany; magnification, x400).

The recombinant virus was screened to confirm its genetic stability. The recombinant virus was passaged for 15 generations, and viral genomes were extracted every 2-3 generations. PCR was performed to amplify the attachment gene of rGTPV-OMP25.

Evaluation of recombinant protein by western blotting. The recombinant virus-infected LT/SFF cells were harvested when 90\% CPE was observed, cells were subsequently freezed/thawed three times and the cellular debris was pelleted. Lysis buffer [60 mM Tris- $\mathrm{HCl}$ ( $\mathrm{pH} 7.1), 1 \mathrm{mM}$ $\mathrm{MgCl}_{2}, 0.05 \% \mathrm{NP} 40$, and $20 \mu \mathrm{g}$ DNase $\left.\mathrm{ml}^{-1}\right]$ was added to lyse the cells. Samples were centrifuged at $350 \mathrm{x} g$ for $5 \mathrm{~min}$ at room temperature to remove the supernatant. Supernatants were mixed with an equal volume of $2 \mathrm{X}$ sample buffer [0.1 M Tris- $\mathrm{HCl}$ (pH 6.8), 4\% sodium dodecylsulfate, $20 \%$ glycerol, $12 \%$ 2-mercaptoethanol and bromophenol blue] and boiled for $10 \mathrm{~min}$. The protein concentrations were determined by a bicinchoninic acid assay. Samples $(25 \mu \mathrm{g})$ were separated by $12.5 \%$ SDS-PAGE and subsequently transferred to nitrocellulose membranes. Membranes were blocked in blocking solution [5\% nonfat milk in Tris-buffered saline Tween-20 (TBST)] for $1 \mathrm{~h}$ at room temperature, and incubated with Brucella-vaccinated sheep serum (Center of Chinese Disease Prevention and Control, Beijing, China; 1:300) diluted in blocking solution at $37^{\circ} \mathrm{C}$ for $1 \mathrm{~h}$. Following three 10-min washes with TBS with Tween-20 (20 mM Tris-HCl, pH 7.6, $150 \mathrm{mM} \mathrm{NaCl,} \mathrm{0.1 \%} \mathrm{Tween-20),}$ membranes were incubated with horseradish peroxidase (HRP)-conjugated rabbit anti-sheep immunoglobulin G (IgG) secondary antibodies (1:5,000; cat. no. SE134; Beijing Solarbio Science \& Technology Co., Ltd., Beijing, China) for $1 \mathrm{~h}$ in $5 \%$ milk/TBST at room temperature. Membranes were washed again and bands were visualized using SuperSignal West Femto Maximum Sensitivity Substrate (Tiangen Biotech Co., Ltd., Beijing, China) according to the manufacturer's protocol.

Immunogenicity of rGTPV expressing OMP25. The levels of OMP25 and GTPV were determined in the sera of mice by ELISA Quantikine Mouse kit (Elabscience Biotechnology, Inc., Houston, TX, USA; cat. no. E-EL-M0692c). The OMP25 protein served as the coating antigen and was diluted with carbonate buffer to 1:500, 1:600 and 1:400. Following this, $100 \mu \mathrm{l}$ was added to the micro-ELISA strip plates for $1 \mathrm{~h}$ at $37^{\circ} \mathrm{C}$ and coated overnight at $4^{\circ} \mathrm{C}$. Strips were washed by filling each well with wash solution $(400 \mu \mathrm{l})$ three times, adding $150 \mu \mathrm{l}$ blocking solution (Sangon Biotech Co. Ltd., Shanghai, China) to each well, washing three times, adding $100 \mathrm{ml}$ test serum (test serum from mice that immunized with OMP25 protein; 1:40) to each well for $1 \mathrm{~h}$ at $37^{\circ} \mathrm{C}$, adding $100 \mathrm{ml}$ sheep anti-mouse HRP-conjugated $\mathrm{IgG}$ secondary antibody (1:5,000; cat. no. ab6808; Abcam, Cambridge, UK) for $1 \mathrm{~h}$ in $5 \%$ milk/TBST to each well for $1 \mathrm{~h}$ at $37^{\circ} \mathrm{C}$, adding $100 \mu \mathrm{l}$ fresh chromogen solution (TransGen Biotech Co., Ltd.) to each well, and finally incubating for $15 \mathrm{~min}$ at $37^{\circ} \mathrm{C}$. Similarly, purified GTPV vaccine strains were used as a coating antigen, and test serum (1:40) was used as a primary antibody. PBS-treated cells served as a control.

Histopathological studies. Histopathological studies were performed as previously described (13). Samples of the liver and kidney and from mice immunized with rGTPV were fixed in $10 \%$ neutral buffered formalin solution at necropsy for 3 days at room temperature. They were dehydrated, embedded in paraffin, and cut into 5-7- $\mu \mathrm{m}$ sections, which were subsequently stained with hematoxylin and eosin for $2 \mathrm{~h}$ at room temperature. Pathological sections were observed using a Motic ordinary light microscope (Motic BA310 DIGITAL; Motic Incorporation, Ltd., Causeway Bay, Hong Kong, China; magnification, $\mathrm{x} 400$ ).

Statistical analysis. Antibody response and cytokine production are expressed as the mean \pm standard deviation of the mean of three independent experiments. Two-tailed 
one-way analysis of variance followed by Bonferroni's post hoc pairwise comparison was used to assess the differences between groups. Statistical comparisons between different groups were conducted using SPSS 17.0 software (SPSS, Inc., Chicago, IL, USA). $\mathrm{P}<0.05$ was considered to indicate a statistically significant difference.

\section{Results}

rGTPV G14-STV44-55 strain transfer vector. The GTPVspecific primers amplified fragments of $2400 \mathrm{bp}$, as expected, that were visualized on a $1 \%$ agarose gel using ethidium bromide staining. Sequence analysis demonstrated 99.54\% homology between the TK gene and GTPV G20-LKV strain (14).

The pLSEG plasmid was digested with XhoI and NotI restriction enzymes, and the reporter gene GPT of $1,200 \mathrm{bp}$ was obtained, as expected, including E. coli. xgpt (459 bp) and the upstream VVP7.5 promoter gene, indicating that a complete construct had been generated. After insertion into the PBS-T vector, transformation and confirmation were performed.

The Brucella OMP25 gene was amplified by PCR; yielding fragments had sizes of $\sim 642 \mathrm{bp}$. PCR fragments derived from the genomic DNA of Brucella were digested and ligated to the $X h o I$ restriction sites of the transfer plasmid pGM-TK-I1L-GPT, to construct the recombinant vector pGM-TK-I1L-GPT-OMP25 (Fig. 1).

Construction of rGTPV expressing OMP25 of Brucella. Recombinant viruses were screened in LT and SFF cells until a CPE was observed. Viruses were tested for genetic stability at defined intervals after repeated passages. In the present study, in a total of 10 generations, viral genomes were extracted every 2-3 generations, and PCR was performed using customized primers to yield amplified fragments with sizes of $642 \mathrm{bp}$.

The transfection product rGTPV-OMP25 was inoculated into confluent SFF and LT cells that were cultured in screening solution for $24 \mathrm{~h}$, and then transfected cells were observed daily for 14 days by microscopy. With the extension of the infection time by rGTPV, the cytopathic phenomenon gradually increased (Fig. 2). The electron microscope examination demonstrated that the viral particles had an oval or round appearance, and brick-shaped virion were identified in cytoplasm (Fig. 2).

The recombinant virus was passaged for 15 generations, and the viral genome was extracted every 2-3 generations; the genetic stability of rGTPV was confirmed by PCR (Fig. 3).

Analysis of recombinant expression vectors by western blotting. To confirm that the OMP25 was expressed in the recombinant vector, whole cell extracts of LT/SFF cells transfected with the GTPV or rGTPV strains were separated by electrophoresis. A band with a molecular mass of $25 \mathrm{kDa}$ was present in rGTPV G14-STV44-55-OMP25 and GTPV G14-STV44-55; however, was missing in untransfected SFF cells. Furthermore, when western blot analysis of the expressed protein was performed using an anti-native Brucella serum, the absence of OMP25 in rGTPV G14-STV44-55-OMP25 was confirmed (Fig. 4).

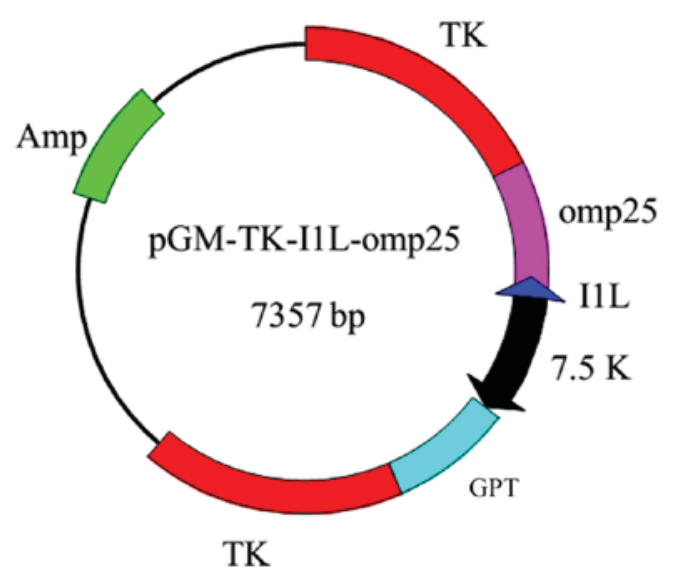

Figure 1. Schematic diagram of the recombinant goat pox virus vector. TK, thymidine kinase; GPT, guanine phosphoribosyl-transferase.

Immunogenicity of rGTPV expressing OMP25. The levels of OMP25 and GTPV in the sera of mice was measured at $450 \mathrm{~nm}$ using a microtiter plate reader. Compared with the control group, OMP25 levels were significantly upregulated in the OMP25 group. However, the rGTPV-OMP25 group exhibited reduced OMP25 levels compared with the OMP25 group (Fig. 5A). GTPV levels were significantly upregulated in the GTPV group compared with the control group; however, there were no significant differences between the GTPV and rGTPV-OMP25 groups (Fig. 5B).

rGTPV-OMP25 is attenuated in BALB/c mice. The liver and kidney are important organs that harbor rGTPV during infection. To assess whether the increased bacterial burden in mice altered liver and kidney pathology, the number and area of granulomas in these organs were determined. As presented in Fig. 6, the mice did not exhibit alterations in liver granulomas after rGTPV infection, indicating that the recombinant vaccine did not cause visible damage to the liver or kidney.

\section{Discussion}

Brucellosis is a zoonotic disease caused by bacteria of the genus Brucella (1), which may lead to substantial economic loss and serious public health problems. Brucellosis will continue to be an important public health concern as long as natural reservoirs exist. Traditional Brucella vaccines, particularly the attenuated vaccine, serve important roles in regulating the disease; however, limitations, including potential problems with safety and other side effects, remain a principal concern. Nevertheless, Brucella remains to be eradicated, and vaccines remain the primary means of treatment, in addition to the most economical means of prevention and control.

OMPs are types of proteins that are common to bacteria. They maintain the stability of the bacterial outer membrane and serve an important role in interactions with the host. OMP25 is the most important Brucella OMP that serves a critical role in maintaining the structural stability of the bacteria. Additionally, OMP25 is a Brucella virulence-associated gene (15).

GTPV vaccine vectors have a large genome structure, which may accommodate large exogenous genes and have a 

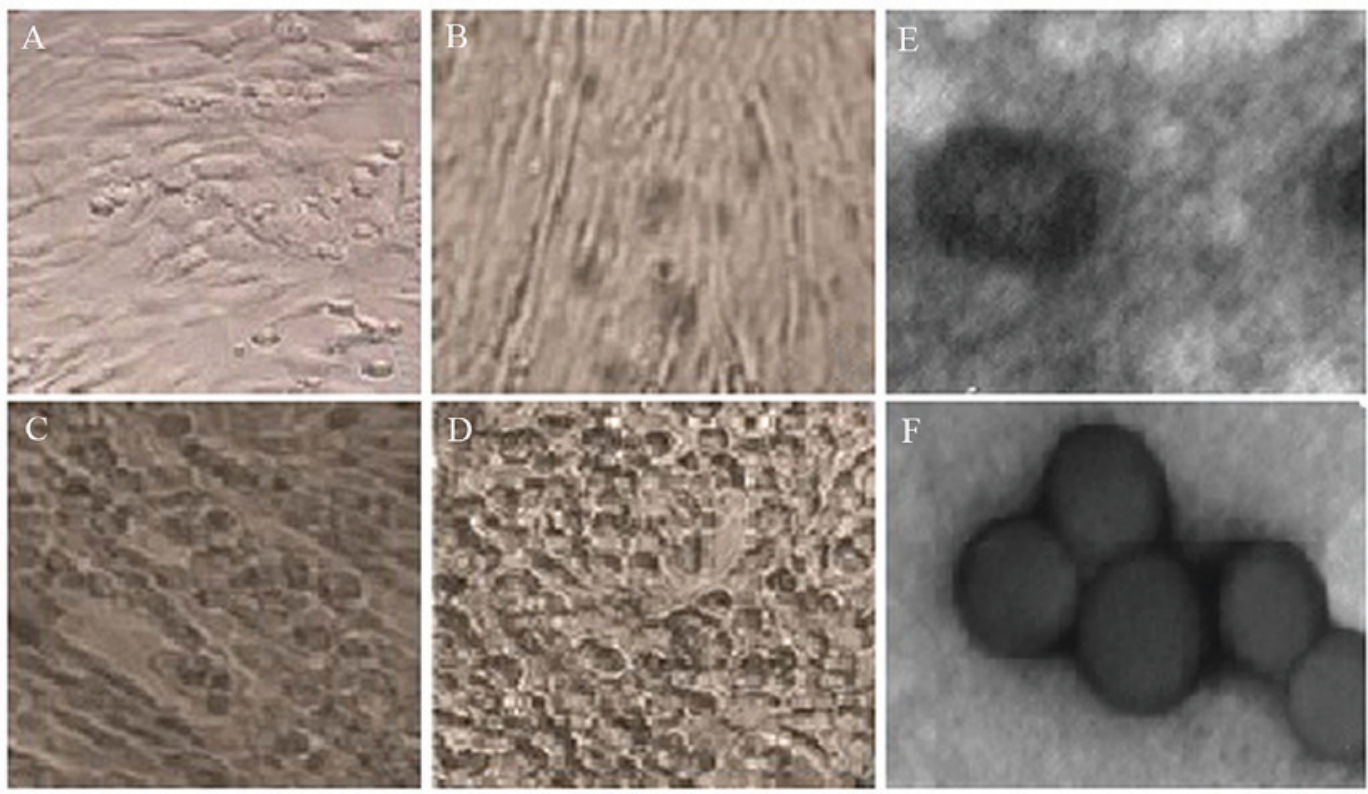

Figure 2. Screening of rGTPV G14-STV44-55-OMP25. The recombinant vector rGTPV-OMP25 was screened in SFF cells. (A) Blank control SFF cells. GTPV-transfected SFF cells examined on days (B) 5, (C) 8 and (D) 14. Images were taken at different perspectives in (E) and (F). Electron microscope examination of rGTPV-OMP25. Magnification, x100,000. GTPV, goat pox virus; rGTPV, recombinant goat pox virus; SFF, sheep fetal fibroblast; LT, lamb testis; OMP25, outer membrane protein 25.

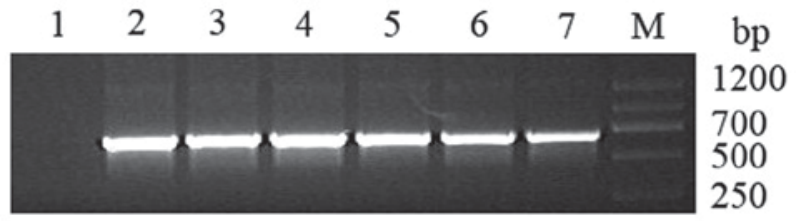

Figure 3. Stability of rGTPV-outer membrane protein 25 . The recombinant vector was passaged for 10 generations, and the viral genome was extracted every 2-3 generations. Polymerase chain reaction was performed using designed primers for amplification of the attachment gene of rGTPV. Lane 1, negative control; lane 2, sample from the third generation; lane 3, sample from the fifth generation; lane 4, sample from the seventh generation; lane 5, sample from the eighth generation; lane 6, sample from the tenth generation; lane 7, sample from the fifteenth generation; lane M, DNA marker; rGTPV, recombinant goat pox virus.

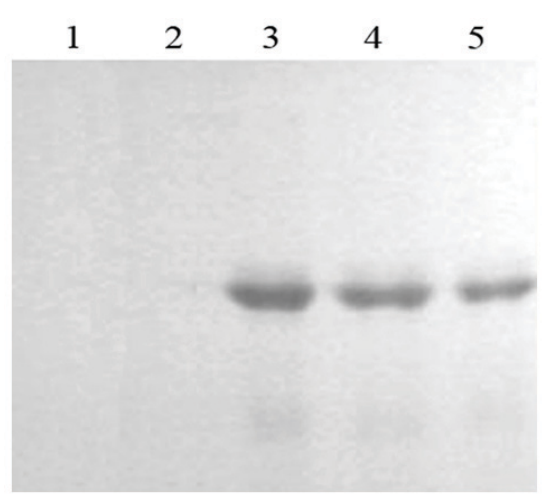

Figure 4. Western blot analysis of the recombinant protein. Lanes 1-3: Cell lysates were separated by SDS-PAGE and incubated with sera from Brucella melitensis-infected mice by western blotting. Lane 1, SFF cells; lane 2, GTPV G14-STV44-55-infected SFF cells; lane 3, rGTPV G14-STV44-55-omp25 infected SFF cells. Lanes 4-5, purified expression OMP25 protein was separated by SDS-PAGE and incubated with sera from rGTPV-OMP25-infected mice (lane 4) or Brucella melitensis-infected mice (lane 5). GTPV, goat pox virus; rGTPV, recombinant goat pox virus; SFF, sheep fetal fibroblast; OMP25, outer membrane protein 25.
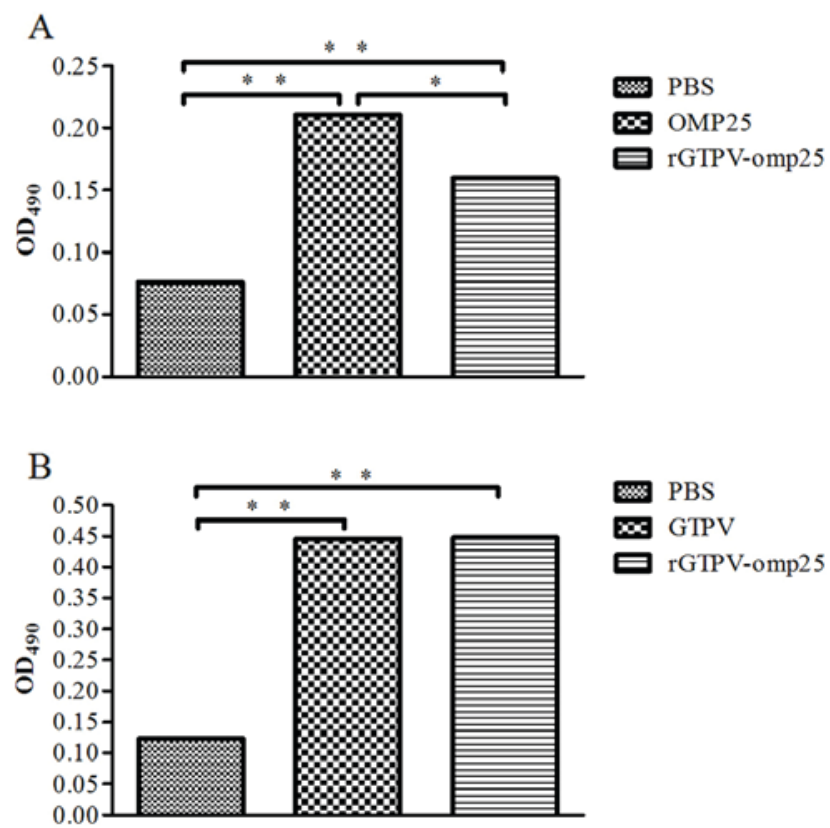

Figure 5. Detection of OMP25 and GTPV of Brucella-melitensis- or rGTPV-OMP25-infected mice. OD value of (A) OMP25 and (B) GPTV in the sera of mice. Data are presented as the mean \pm standard deviation. "P $<0.05,{ }^{* *} \mathrm{P}<0.01$. OD, optical density; GTPV, goat pox virus; rGTPV, recombinant goat pox virus; OMP25, outer membrane protein 25 .

good safety profile. Importantly, it may replicate by itself and express exogenous genes following entry into the body (16). Previously, the use of GTPV as a vaccine vector has been reported in China and abroad, and numerous ruminant mammal protective antigens have been expressed in the GTPV vaccine vector expression system, which have demonstrated promising results $(17,18)$. The present study selected VVI1L promoter-expressed exogenous genes as VVI1L is a 

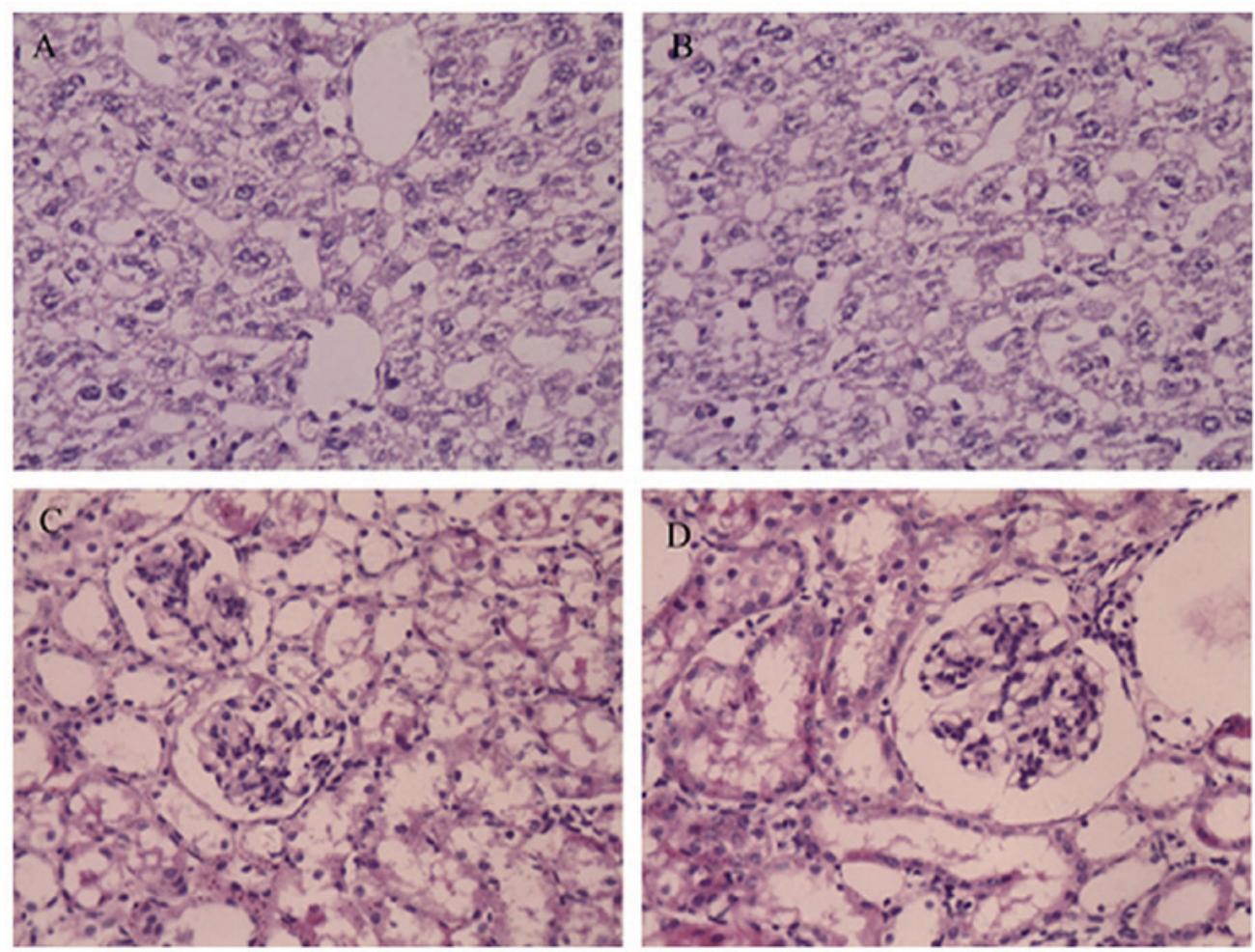

Figure 6. Recombinant GTPV does not affect liver and kidney granuloma formation. Liver tissues from (A) rGTPV-OMP25-infected and (B) control mice. Kidney tissues from (C) rGTPV-OMP25-infected and (D) control mice. Hematoxylin and eosin staining was performed. Magnification, x40. GTPV, goat pox virus; rGTPV, recombinant goat pox virus; OMP25, outer membrane protein 25.

strong promoter, as its activity is 10 -fold greater than that of VVP7.5 (10,12).

The present study demonstrated that the immunogenicity of rGTPV-OMP25 is weak. A reason for this may be that the immunological effect of rGTPV-OMP25 in non-ruminants is weaker compared with ruminants. Therefore, rGTPV-OMP25 will be further evaluated in goats and sheep. Furthermore, Brucella-associated genes expressed by rGTPV may additionally contribute to reduced immunogenicity.

In conclusion, in the present study, an overexpression vector for the Brucella OMP OMP25 was constructed and identified a robust. The construct was recombined with GTPV, and the immunostimulatory activity was confirmed. This novel vaccine may aid efforts to prevent brucellosis and goat pox infections, and may yield novel insights for research efforts aimed at generating Brucella and goat pox bivalent vaccines.

\section{Acknowledgements}

Not applicable.

\section{Funding}

The present study was supported by the National key Research and Development Program of China (grant no. 2017YFD0500304), the Training Program for Excellent Young Teachers Colleges and Universities of Corps (grant no. CZ027202), the National Natural Science Foundation of China (grant nos. 31860691 and 31602080), the Youth Science and technology innovation leading talent program of
Corps (grant no. 2017CB002), the Foundation of the Technology Department of Henan Province (grant no. 172102310335), and the Shihezi University international science and technology cooperation promotion plan (grant no. GJHZ201709).

\section{Availability of data and materials}

The datasets used and/or analyzed during the current study are available from the corresponding author on reasonable request.

\section{Authors' contributions}

HZ, ZG and CC designed the experiments. ZS, LL, YL, FW and $\mathrm{QF}$ performed the experiments. ZL, PW, YR and YZ analyzed the data. ZS, LL and ZL wrote the manuscript. HZ and $Z \mathrm{~L}$ revised the manuscript. All authors read and approved the final manuscript.

\section{Ethics approval and consent to participate}

The present study was approved by the Institutional Committee of Post-Graduate Studies and Research at Shihezi University.

\section{Patient consent for publication}

Not applicable.

\section{Competing interests}

The authors declare they have no competing interests. 


\section{References}

1. Bercovich Z: The use of skin delayed-type hypersensitivity as an adjunct test to diagnose brucellosis in cattle: A review. Vet Q 22: 123-130, 2000

2. Wang Z and Wu Q: Research progress in live attenuated Brucella vaccine development. Curr Pharm Biotechnol 14: 887-896, 2013.

3. Cloeckaert A, de Wergifosse P, Dubray G and Limet J: Identification of seven surface-exposed Brucella outer membrane proteins by use of monoclonal antibodies: Immunogold labeling for electron microscopy and enzyme-linked immunosorbent assay. Infect Immun 58: 3980-3987, 1990.

4. Shevtsov A, Tarlykov P, Zholdybayeva E, Shevtsova E, Momynkulov D, Sytnik I, Karibaev T, Chsherbakov A and Momynaliev K: Draft genome sequence of the live vaccine strain Brucella abortus 82. Genome Announc 1: e01101-01113, 2013.

5. Schurig GG, Sriranganathan N and Corbel MJ: Brucellosis vaccines: Past, present and future. Vet Microbiol 90: 479-496, 2002.

6. Diallo A, Minet C, Berhe G, Le Goff C, Black DN, Fleming M, Barrett T, Grillet C and Libeau G: Goat immune response to capripox vaccine expressing the hemagglutinin protein of peste des petits ruminants. Ann N Y Acad Sci 969: 88-91, 2002.

7. Venkatesan G, Balamurugan V, Yogisharadhya R, Kumar A and Bhanuprakash V: Differentiation of sheeppox and goatpox viruses by polymerase Chain reaction-restriction fragment length polymorphism. Virol Sin 27: 353-359, 2012.

8. Zhao Z, Wu G, Zhu X, Yan X, Dou Y, Li J, Zhu H, Zhang Q and Cai X: RNA interference targeting virion core protein ORF095 inhibits Goatpox virus replication in Vero cells. Virol J 9: 48, 2012.

9. Mackett M, Smith GL and Moss B: Vaccinia virus: A selectable eukaryotic cloning and expression vector. Proc Natl Acad Sci USA 79: 7415-7419, 1982.
10. Liu X, Kremer M and Broyles SS: A natural vaccinia virus promoter with exceptional capacity to direct protein synthesis. J Virol Methods 122: 141-145, 2004.

11. Falkner FG and Moss B: Escherichia coli gpt gene provides dominant selection for vaccinia virus open reading frame expression vectors. J Virol 62: 1849-1854, 1988.

12. Fick WC and Viljoen GJ: Identification and characterisation of an early/late bi-directional promoter of the capripoxvirus, lumpy skin disease virus. Arch Virol 144: 1229-1239, 1999.

13. Chen H, Li Y, Li Z, Shi J, Shinya K, Deng G, Qi Q, Tian G, Fan S, Zhao $\mathrm{H}$, et al: Properties and dissemination of $\mathrm{H} 5 \mathrm{~N} 1$ viruses isolated during an influenza outbreak in migratory waterfowl in western China. J Virol 80: 5976-5983, 2006.

14. Feng W, Zhiru G, Chuangfu C and Jun Q: Construction of the recombinant goat pox virus expressing OMP 25 gene of $\mathrm{B}$. melitensis. Prog Veterinary Med 29: 6-9, 2008.

15. Goel D, Rajendran V, Ghosh PC and Bhatnagar R: Cell mediated immune response after challenge in Omp25 liposome immunized mice contributes to protection against virulent Brucella abortus 544. Vaccine 31: 1231-1237, 2013.

16. Gershon PD and Black DN: A comparison of the genomes of capripoxvirus isolates of sheep, goats, and cattle. Virology 164: 341-349, 1988.

17. Tulman ER, Afonso CL, Lu Z, Zsak L, Sur JH, Sandybaev NT, Kerembekova UZ, Zaitsev VL, Kutish GF and Rock DL: The genomes of sheeppox and goatpox viruses. J Virol 76: 6054-6061, 2002.

18. Berhe G, Minet C, Le Goff C, Barrett T, Ngangnou A, Grillet C, Libeau G, Fleming M, Black DN and Diallo A: Development of a dual recombinant vaccine to protect small ruminants against peste-des-petits-ruminants virus and capripoxvirus infections. J Virol 77: 1571-1577, 2003. 